\title{
Long intergenic non-coding RNA, regulator of reprogramming (LINC-ROR) over-expression predicts poor prognosis in renal cell carcinoma
}

\author{
Manal S. Fawzy ${ }^{1,2}$, Eman A. Toraih ${ }^{3,4}$, Aya El-Wazir ${ }^{3,4}$, Marwa M. Hosny ${ }^{1}$, Dahlia I. Badran ${ }^{1}$, Amr El Kelish ${ }^{5}$
}

\author{
${ }^{1}$ Department of Medical Biochemistry and Molecular Biology, Faculty of Medicine, \\ Suez Canal University, Ismailia, Egypt \\ 2Department of Biochemistry, Faculty of Medicine, Northern Border University, Arar, \\ Saudi Arabia \\ ${ }^{3}$ Genetics Unit, Department of Histology and Cell Biology, Faculty of Medicine, \\ Suez Canal University, Ismailia, Egypt \\ ${ }^{4}$ Center of Excellence of Molecular and Cellular Medicine, Suez Canal University, \\ Ismailia, Egypt \\ ${ }^{5}$ Botany Department, Faculty of Science, Suez Canal University, Ismailia, Egypt
}

Submitted: 19 April 2018; Accepted: 14 July 2018

Online publication: 17 May 2019

Arch Med Sci 2021; 17 (4): 1016-1027

DOI: https://doi.org/10.5114/aoms.2019.85201

Copyright (c) 2019 Termedia \& Banach

\section{Abstract}

Introduction: Long intergenic non-coding RNA, regulator of reprogramming (LINC-ROR) is a newly identified cytoplasmic long non-coding RNA (InCRNA) implicated in cell longevity and apoptosis. We aimed in the current work for the first time to investigate the association of the expression profiles of LINC-ROR and three stem-related transcriptional factors with clinicopathological data and their impact on renal cell carcinoma (RCC) progression in a sample of RCC patients.

Material and methods: Expression levels of LINC-ROR and stemness-related factors: SOX2, NANOG, and POU5F1 were detected in 60 formalin-fixed, paraffin-embedded tissues, and their paired adjacent non-cancer tissues ( $n=60$ ) by using real-time qRT-PCR analysis. Additionally, the expression profiles were compared with the available clinicopathological features.

Results: The genes studied were markedly up-regulated in RCC (medians and interquartile ranges were 30.3 (1.84-235.5), 10.2 (1.84-53.9), 5.39 (0.94-23.5), and 12.5 (1.61-43.2) for LINC-ROR, SOX2, NANOG, and POU5F1, respectively) relative to paired non-cancer tissue. High expression levels were associated with poor prognosis in terms of tumour undifferentiation (for LINC-ROR, SOX2, and NANOG), lymph node infiltration (for SOX2), postoperative recurrence (for $L I N C-R O R$ and SOX2), and shorter overall survival (OS) and progression-free survival (for all genes studied). The best curve for OS prediction was constructed with LINC-ROR data (area under the receiver operating characteristic curve (AUC) $=0.804$ at a cut-off value of 72.7 , sensitivity $78.9 \%$, and specificity $80.5 \%$ ).

Conclusions: Collectively, aberrant LINC-ROR and pluripotent gene expression may be recognised as prognostic markers for RCC. Future functional studies are highly recommended to validate the study findings.

Key words: renal cell carcinoma, long intergenic non-coding RNA, regulator of reprogramming, SOX2, NANOG, POU5F1.

\author{
Corresponding authors: \\ Manal S. Fawzy PhD, MD \\ Department of Medical \\ Biochemistry \\ and Molecular Biology \\ Faculty of Medici \\ Suez Canal University \\ 41522 Ismailia, Egypt \\ Phone: +(2) 01008584720 \\ E-mail: \\ manal2_khashana@ymail.com
}

Eman A. Toraih MD, PhD

Department of Histology

and Cell Biology

Genetics Unit

Faculty of Medicine

Suez Canal

University

41522 Ismailia, Egypt

Phone: +(2) 01008550681

E-mail:

emantoraih@gmail.com 


\section{Introduction}

Renal cell carcinoma (RCC), which arises from renal tubular epithelial cells, accounts for $2-3 \%$ of all cancers worldwide [1]. There has been a global rise in RCC incidence in past decades, and up to $35 \%$ of RCC patients present with metastasis [2]. Therefore, discovering biomarkers for early detection and better prognostic stratification of RCC has emerged as a noteworthy target for future investigations.

According to the World Health Organisation (WHO), there are three major histopathological RCC types: clear cell RCC (ccRCC), papillary cell RCC (pcRCC), and chromophobe RCC (chRCC) [3]. Each RCC subtype is known to display unique pathological characteristics, distinct genetic alterations, and different disease outcomes [1]. The interplay of the genetic mechanisms that affect RCC pathogenesis is still poorly understood. The use of integrated molecular analyses enables a deeper understanding of the RCC molecular landscape, resulting in the development of more effective targeted cancer therapies as an extension of existing modalities $[4,5]$.

Cancer has been found to histologically mimic embryonic tissue [6]. Aggressive cancer cells exhibit phenotypic traits remarkably similar to those of pluripotent stem cells; both have high proliferation rates and a substantial ability to self-renew by bypassing senescence [7]. Accumulating evidence has demonstrated the convergence of tumourigenic signalling and developmental pathways [8]. Since the introduction of induced pluripotent stem cell (iPSC) generation mediated by transcription factor (TF) reactivation, studies have been directed towards identifying the differential expression profiles altered during pathological nuclear reprogramming in cancer [7]. SOX2, NANOG, and POU5F1 (also known as OCT3/4) are three pluripotency-associated TFs that are well known to be up-regulated in various cancer types through modulation of apoptotic signalling pathways [9]. Notably, modifying the expression of pluripotency-associated genes in animal models has generated epithelial-derived tumours in multiple tissues with altered spatial and temporal gene expression [10].

Recent evidence has suggested complex networking between TFs, chromatin organisation, and non-coding RNAs that regulate cell fate and differentiation. Several long intergenic non-coding RNAs (lincRNAs) have also been shown to be associated with pluripotency [11]. LincRNA expression has been shown to be highly correlated with the expression of stem cell-related TFs [12]. Binding sites for pluripotency-related genes have been identified within the promoters of these long non-coding RNAs (IncRNAs) [13]. In vitro silencing of lincRNAs has been shown to induce cells to exit the pluripotent state and altered gene expression patterns [14]. In 2010, Loewer et al. [11] identified lincRNA, regulator of reprogramming (LINC-ROR or ROR) in human fibroblasts and CD34-positive blood cells during iPSC generation. Furthermore, in vitro and in vivo studies have determined the critical role of ROR in modulating cell proliferation, apoptosis, and chemosensitivity in cancer [15-17].

To the best of our knowledge, no clinical studies have been conducted exploring the role of ROR in RCC patients to validate the aforementioned in vitro and in vivo results. In the current study, we aimed to analyse the transcriptomic signature profiling of LINC-ROR and three of the main pluripotency-related genes (SOX2, NANOG, and POU5F1) in RCC patients to assess their clinical utility as prognostic biomarkers.

\section{Material and methods}

Case selection and pathological assessment

In total, 120 formalin-fixed, paraffin-embedded (FFPE) specimens were analysed, including 60 RCC

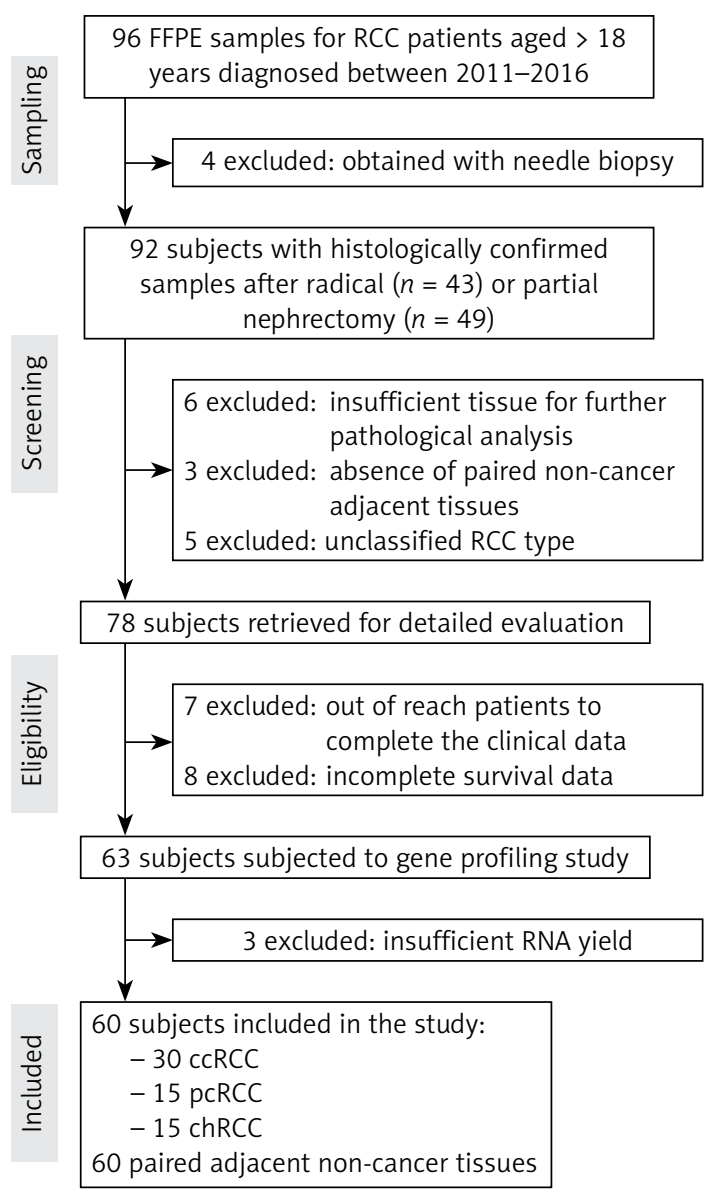

Figure 1. Flow chart of the study population selection.

FFPE - formalin-fixed paraffine-impeded, $R C C$ - renal cell carcinoma, $c C R C C$ - clear cell RCC, $P C R C C$ - papillary cell $R C C$, chRCC - chromophobe RCC. 
samples (30 ccRCC, 15 pcRCC, and 15 chRCC) and their paired adjacent non-cancer tissues. Figure 1 shows the flow chart of the current study case selection. All specimens were obtained after radical or partial nephrectomy, and cases with needle biopsies were excluded. All retrieved cases were archived in the Department of Pathology, Suez Canal University between 2011 and 2016. Patient data were obtained from medical records. Missing data were directly retrieved from the patients. Patient follow-ups were performed until December 2016 and ranged from seven to 32 months. Postoperative recurrence was calculated, and survival times were estimated from the date of nephrectomy until patient death or the endpoint of follow-up. The study was conducted following the ethical and legal standards adopted by the Declaration of Helsinki. Approval was obtained from the Medical Research Ethics Committee of the Faculty of Medicine, Suez Canal University. Consent was directly obtained from patients as a routine measure for sample archiving.

\section{Histopathological examination}

Histological examination, nuclear grading, and tumour staging of RCC samples were based on haematoxylin and eosin staining. Blind assessments were made without prior knowledge, according to the internationally standardised protocols we mentioned in our previous work [18]. Most of the RCC cases were the clear cell type and contained very little delicate vascular stroma. The papillary and chromophobe types also had only a little amount of stroma, and thus it was unlikely that it affected accurate determination of the expression of the genes assessed in this study.

\section{Gene expression profiling}

Total RNA was purified from the FFPE sections using a Qiagen RNeasy FFPE Kit (Cat \# 74404, Qiagen, Hilden, Germany) following the manufacturer's protocol. A short incubation of each sample with proteinase $\mathrm{K}$ at a higher temperature, as recommended, partially reverses formalin crosslinking of the released nucleic acids, improving RNA yield and quality as well as RNA performance in downstream assays. All samples were subjected to treatment with RNase-free DNase I for $2 \mathrm{~h}$ at $37^{\circ} \mathrm{C}$ to eliminate all genomic DNA, including very small DNA fragments that are often present in FFPE samples after prolonged formalin fixation and/or long storage times. At an absorbance ratio of 260/280 nm, RNA concentration and purity were assessed by a Nanodrop ND-1000 spectrophotometer (NanoDrop Technologies, Wilmington, $D E)$, followed by agarose gel electrophoresis check for RNA integrity. The range of the extracted RNA was $(20-65 \mathrm{ng} / \mu \mathrm{l})$. Reverse transcription was performed with a high-capacity cDNA reverse tran- scription kit (Part No. 4374966, Applied Biosystems, Thermo Fisher Scientific, Waltham, MA, USA) using a Mastercycler Gradient Thermocycler (Eppendorf, Hamburg, Germany). Detection of LINCROR, SOX2, NANOG, and POU5F1 gene expression and that of the endogenous control GAPDH was performed using real-time quantitative reverse transcriptase polymerase chain reaction (qRT$P(R)$. TaqMan assays were used for the pluripotent genes (Applied Biosystems, Thermo Fisher Scientific Inc., assay ID Hs01053049_s1 for SOX2, Hs02387400_g1 for NANOG, Hs03005111_g for POU5F1, and Hs02786624_g1 for GAPDH) as previously described in detail [19]. Quantification of LINC-ROR was achieved using SYBR Green with primers for exon 4 (F: GCCTGAGAGTTGGCATGAAT and R: AAAACCTCACTCCCATGTGC) [20]. The Minimum Information for Publication of Quantitative Real-Time PCR Experiments (MIQE) guidelines were followed for all PCR reactions. Gene expression in randomly selected study samples (10\%) was re-evaluated in separate runs to test the reproducibility of the qPCR analysis, and the results showed very close cycle of quantification (Cq) values with low standard deviations.

\section{Statistical analysis}

Data analyses were conducted using the R packages SPSS version 22 and GraphPad Prism version 7. Multivariate analysis was conducted using PCORD version 5.0. The relative expression levels were calculated using the delta-delta threshold d cycle method. The Wilcoxon matched-pair signed-rank test was carried out to compare the expression level between cancer samples and their corresponding adjacent non-cancer tissues. Receiver operating characteristic (ROC) curves and the area under the ROC curves (AUCs) were determined to identify the clinical utility of the expressed genes. Ordination analysis and two-way agglomerative hierarchical clustering were performed for data exploration. Spearman's correlation analysis, chi-square $\left(\chi^{2}\right)$, Fisher's exact, Mann-Whitney $U(\mathrm{MW})$, and Kruskal-Wallis (KW) tests were applied when appropriate. A two-tailed $p$-value was considered significant at values $<0.05$. Binary logistic and linear regression methods (Enter and Stepwise models) were applied to determine predictors for recurrence and overall survival (OS). The influence of covariates, such as age, gender, grade, tumour type, size, and gene profile, on OS was analysed. Kaplan-Meier analysis and the Cox proportional hazard model were performed to assess survival rates.

\section{Results}

\section{Characteristics of the study patients}

The baseline features of RCC patients are shown in Table I. Overall, 21 female and 39 male 
Table I. Clinicopathological characteristics of renal cell carcinoma patients $(n=60)$

\begin{tabular}{|c|c|c|c|c|c|}
\hline Parameter & $N$ & $\%$ & Parameter & $N$ & $\%$ \\
\hline Age: & & & Tumour size: & & \\
\hline$<60$ years & 28 & 46.7 & T1 & 21 & 35.0 \\
\hline$\geq 60$ years & 32 & 53.3 & T2 & 25 & 41.7 \\
\hline Gender: & & & T3 & 14 & 23.3 \\
\hline Female & 21 & 35.0 & Pathological grade: & & \\
\hline Male & 39 & 65.0 & Grade 1 & 9 & 15.0 \\
\hline Histopathological type: & & & Grade 2 & 28 & 46.7 \\
\hline Clear cell RCC & 30 & 50.0 & Grade 3 & 23 & 38.3 \\
\hline Papillary RCC & 15 & 25.0 & Recurrence: & & \\
\hline Chromophobic RCC & 15 & 25.0 & Negative & 44 & 73.3 \\
\hline Tumour location: & & & Positive & 16 & 26.7 \\
\hline Right side & 22 & 36.7 & Progression-free survival: & & \\
\hline Left side & 38 & 63.3 & $\leq 12$ months & 22 & 36.7 \\
\hline Number: & & & $\leq 24$ months & 31 & 51.6 \\
\hline Solitary & 46 & 76.7 & $\leq 36$ months & 7 & 11.7 \\
\hline Multifocal & 14 & 23.3 & Overall survival: & & \\
\hline LN involvement: & & & $\leq 12$ months & 19 & 31.7 \\
\hline Negative & 39 & 65.0 & $\leq 24$ months & 34 & 56.6 \\
\hline Positive & 21 & 35.0 & $\leq 36$ months & 7 & 11.7 \\
\hline
\end{tabular}

Table II. Correlation matrix for the clinicopathological characteristics of renal cell carcinoma patients $(n=60)$

\begin{tabular}{|c|c|c|c|c|c|c|c|c|c|}
\hline Parameter & Age & Gender & No. & Grade & $T$ & LN & $\operatorname{Rec}$ & PFS & os \\
\hline Age & 1.000 & & & & & & & & \\
\hline \multirow[t]{2}{*}{ Gender } & -0.229 & 1.000 & & & & & & & \\
\hline & $(0.078)$ & & & & & & & & \\
\hline \multirow[t]{2}{*}{ No } & -0.218 & -0.008 & 1.000 & & & & & & \\
\hline & $(0.095)$ & $(0.950)$ & & & & & & & \\
\hline \multirow[t]{2}{*}{ Grade } & 0.017 & -0.213 & 0.453 & 1.000 & & & & & \\
\hline & $(0.897)$ & $(0.103)$ & $(<0.001)$ & & & & & & \\
\hline \multirow[t]{2}{*}{$\mathrm{T}$} & -0.074 & -0.205 & 0.491 & 0.447 & 1.000 & & & & \\
\hline & $(0.576)$ & $(0.116)$ & $(<0.001)$ & $(<0.001)$ & & & & & \\
\hline \multirow[t]{2}{*}{ LN } & 0.070 & -0.194 & 0.504 & 0.631 & 0.557 & 1.000 & & & \\
\hline & $(0.597)$ & $(0.137)$ & $(<0.001)$ & $(<0.001)$ & $(<0.001)$ & & & & \\
\hline \multirow[t]{2}{*}{$\operatorname{Rec}$} & -0.180 & -0.111 & 0.202 & 0.460 & 0.189 & 0.348 & 1.000 & & \\
\hline & $(0.169)$ & $(0.400)$ & $(0.122)$ & $(<0.001)$ & $(0.149)$ & $(0.006)$ & & & \\
\hline \multirow[t]{2}{*}{ PFS } & 0.260 & 0.182 & -0.154 & -0.417 & -0.144 & -0.243 & -0.675 & 1.000 & \\
\hline & $(0.045)$ & $(0.164)$ & $(0.241)$ & $(0.001)$ & $(0.271)$ & $(0.061)$ & $(<0.001)$ & & \\
\hline \multirow[t]{2}{*}{ OS } & 0.243 & 0.222 & -0.132 & -0.347 & -0.123 & -0.152 & -0.523 & 0.968 & 1.000 \\
\hline & $(0.061)$ & $(0.088)$ & $(0.314)$ & $(0.007)$ & $(0.350)$ & $(0.248)$ & $(<0.001)$ & $(<0.001)$ & \\
\hline
\end{tabular}

Data are presented as a correlation coefficient ( $P$ value). Spearman's rank correlation was used. No. - number, $T-$ tumour size, $L N-l y m p h$ node, Rec-recurrence, PFS - progression-free survival, OS - overall survival. Bold values are considered statistically significant at $p<0.05$.

RCC patients were included in the study. Their ages ranged from 48 to 79 years (mean \pm standard deviation of $58.6 \pm 6.31)$. Notably, in patients with solitary tumours (76.7\%), the left kidney was more frequently affected (63.3\%). Despite the lack of lymph node metastases in nearly two-thirds of the spec- imens (65\%) and the small tumour sizes (76.7\%), most samples showed moderate/poor differentiation (85\%). Recurrence occurred in approximately one-fourth of patients (26.7\%) after a mean duration of $4 \pm 1.7$ months. Postoperative disease-free survival ranged from 2 to 32 months. The OS rate 
A

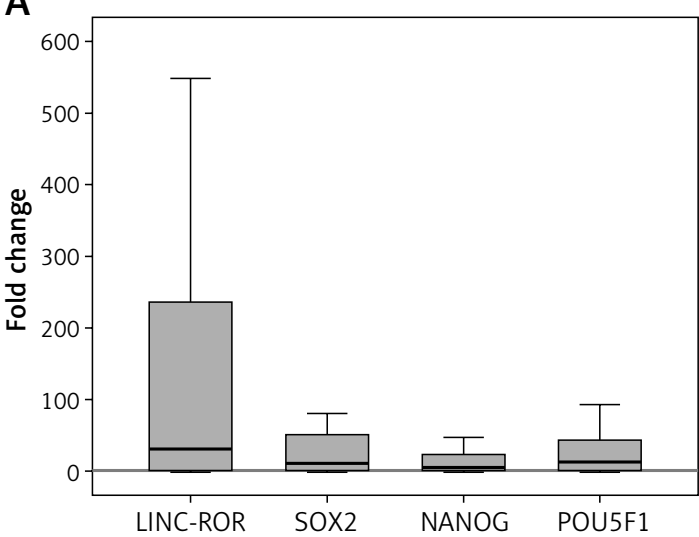

B

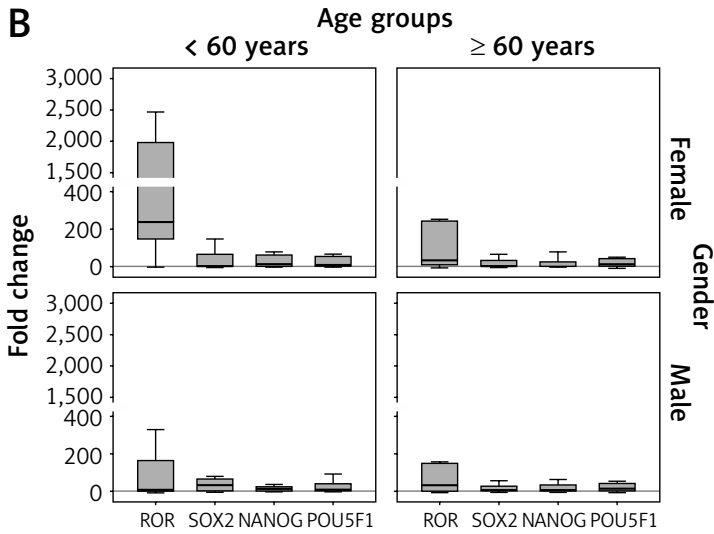

Figure 2. Gene expression of LINC-ROR and pluripotent genes in RCC. The data are presented as the median values. The box defines the upper and lower quartiles ( $25 \%$ and $75 \%$, respectively), and the error bars indicate the upper and lower adjacent limits. Gene expression levels in cancer and normal tissues were normalised to GAPDH expression and were calculated using the delta-delta CT method $[=2(-\Delta \Delta C T)]$ in comparison with normal renal tissues. The red line represents the expression level of normal renal tissues (equivalent to 1 ). The Wilcoxon matched-pair signed-rank test was used. All $p$-values were $<0.001$. A - Overall analysis. B - Stratified analysis by age and gender

was $68.3 \%$ in the first year and $11.7 \%$ in the second year, with a mean \pm SD of $11.4 \pm 4.9$ months. The correlation matrix showed that tumour grade was positively correlated with tumour number $(r=0.453, p<0.001)$, larger tumour size $(r=0.447$, $p<0.001)$, lymph node metastasis $(r=0.631$, $p<0.001)$, and post-operative recurrence $(r=0.460$, $p<0.001)$ but negatively correlated with progression-free survival (PFS; $r=-0.417, p=0.001$ ) and OS ( $r=-0.347, p=0.007)$. Tumour recurrence was moderately correlated with lymphadenopathy $(r=0.348, p=0.006)$. Patient age showed a weak association with PFS $(r=0.260, p=0.045)$ (Table II).

\section{Gene expression analyses}

Gene expression profiling demonstrated overexpression of the LINC-ROR, SOX2, NANOG, and
POU5F1 genes in tumour specimens compared with those in their paired non-cancer tissues (all $p<0.001)$. Medians and interquartile ranges of the relative expression levels in RCC were 30.3 (1.84235.5) for LINC-ROR, 10.2 (1.84-53.9) for SOX2, 5.39 (0.94-23.5) for NANOG, and 12.5 (1.61-43.2) for POU5F1 (Figure 2). Stratified analyses by age and gender showed similar up-regulation (all $p$-values $<0.001$ ), with marked over-expression of LINC-ROR in female patients less than 60 years of age at disease onset. The correlation matrix showed a significant positive relationship between LINC-ROR and both SOX2 $(r=0.280, p=0.045)$ and NANOG $(r=0.312, p=0.015)$.

\section{Prognostic value of gene expression analyses}

Univariate analysis revealed marked associations between LINC-ROR, SOX2, and NANOG expression levels and undifferentiated tumours ( $p=0.010,0.046$, and 0.042, respectively). SOX2 up-regulation was linked to lymph node infiltration $(p=0.047)$. Moreover, patients with higher LINC-ROR and SOX2 levels experienced post-operative recurrence $(p=0.003$ and 0.035$)$. The expression of all genes presented significant associations with shorter PFS and low OS (all $p<0.05$ ) (Table III). Similar results were found in correlation analyses between gene expression levels and the clinicopathological findings (Table IV).

The prognostic performance of the four genes in predicting OS in RCC patients was assessed using ROC analyses (Figure 3). In single-gene analyses, LINC-ROR data elicited the best curve (AUC $=0.804$ at a cut-off value of 72.7 ; sensitivity $78.9 \%$, specificity $80.5 \%$ ) followed by NANOG (AUC $=0.748$ at a cut-off value 11.2 ; sensitivity $68.4 \%$, specificity $75.6 \%$ ) and SOX2 (AUC = 0.714 at a cut-off value 25.5 ; sensitivity $73.3 \%$, specificity $75.6 \%$ ), whereas POU5F1 had an AUC of 0.632 at a cut-off value of 15.2 , a sensitivity of $52.6 \%$, and a specificity of $56.1 \%$. Despite the good performance of the combined gene analyses, with AUC values ranging from 0.675 to 0.755 , the AUC of LINC-ROR remained the best (Table V).

Logistic regression analyses revealed that both lymph node infiltration and LINC-ROR expression acted as recurrence predictors in RCC patients $(p=0.007$ and 0.047, respectively; Table VI). Furthermore, linear regression analyses demonstrated that LINC-ROR was an independent predictor for OS $\left(R=0.443, R^{2}=0.192, p<0.001\right)$.

\section{Survival analysis}

Analyses of different influencing factors on the survival time of RCC patients are demonstrated in Table VII. Cox regression showed that LINC-ROR, POU5F1, and recurrence were risk factors that predicted OS (hazard ratio $(H R)=4.21,95 \%$ confi- 
Long intergenic non-coding RNA, regulator of reprogramming (LINC-ROR) over-expression predicts poor prognosis in renal cell carcinoma

Table III. Univariate analysis for association between gene profile and the clinicopathological features in RCC patients

\begin{tabular}{|c|c|c|c|c|c|c|c|c|c|}
\hline \multirow[t]{2}{*}{ Parameter } & & \multicolumn{2}{|c|}{ LINC-ROR } & \multicolumn{2}{|l|}{ sox2 } & \multicolumn{2}{|l|}{ NANOG } & \multicolumn{2}{|c|}{ POU5F1 } \\
\hline & & Expression & $P$-value & Expression & $P$-value & Expression & $P$-value & Expression & $P$-value \\
\hline Age: & & & 0.450 & & 0.178 & & 0.563 & & 0.947 \\
\hline$<60$ years & 28 & $15(1.3-236)$ & & $28.6(1.8-65)$ & & $10(0.9-23.5)$ & & $7.9(1.9-44.1)$ & \\
\hline$\geq 60$ years & 32 & $33(4.8-214)$ & & $7.19(1.3-34)$ & & $3.9(0.7-30.5)$ & & $14(1.4-41.3)$ & \\
\hline Gender: & & & 0.060 & & 0.62 & & 0.871 & & 0.625 \\
\hline Female & 21 & $39(11.2-575)$ & & $8.6(1.7-40.9)$ & & $4.7(1.3-38)$ & & $14.3(2.8-46)$ & \\
\hline Male & 39 & $12(1.3-152)$ & & $12(1.7-56.8)$ & & $5.6(0.5-23.1)$ & & $12.3(1.5-42)$ & \\
\hline HPD: & & & 0.737 & & 0.818 & & 0.302 & & 0.906 \\
\hline $\mathrm{cCRCC}$ & 30 & $18(1.5-149)$ & & $8.0(1.9-45)$ & & $4.8(0.2-15)$ & & $14(1.5-45)$ & \\
\hline $\mathrm{pRCC}$ & 15 & $30(0.5-654)$ & & $25(1.0-65)$ & & $15(1.6-61)$ & & $12(0.7-42)$ & \\
\hline chRCC & 15 & $36(2.8-236)$ & & $12(1.7-61)$ & & $4.7(02-14)$ & & $10(1.7-43)$ & \\
\hline Side: & & & 0.914 & & 0.742 & & 0.730 & & 0.137 \\
\hline Right & 22 & $21(2.2-169)$ & & $14(3.1-56)$ & & $4.6(0.4-50)$ & & $3.8(1.2-34)$ & \\
\hline Left & 38 & $36(1.6-239)$ & & $10(1.6-16)$ & & $5.9(1.3-16)$ & & $16(2.3-44)$ & \\
\hline Number: & & & 0.442 & & 0.144 & & 0.302 & & 0.479 \\
\hline Unifocal & 46 & $27(2.2-165)$ & & $9.4(0.4-45)$ & & $4.9(0.4-20.9)$ & & $13(1.5-44)$ & \\
\hline Multifocal & 14 & $34(1.4-985)$ & & $17(3.9-85)$ & & $13(1.8-42.6)$ & & $73(1.6-23)$ & \\
\hline Grade: & & & $0.010^{\star}$ & & $0.046^{\star}$ & & $0.042^{*}$ & & 0.435 \\
\hline Grade 1 & 9 & $3.3(0.6-105)$ & & $1.3(0.16-61)$ & & $6.5(0.004-21)$ & & $12.3(1.7-73)$ & \\
\hline Grade 2 & 28 & $13(1.8-112)$ & & $7.1(0.63-30)$ & & $2.8(0.21-9.3)$ & & $9.9(1.4-32.2)$ & \\
\hline Grade 3 & 23 & $235(7.8-898)$ & & $35.7(3.9-65)$ & & $12.3(2.0-61)$ & & $15(2.5-44.3)$ & \\
\hline $\mathrm{T}:$ & & & 0.071 & & 0.063 & & 0.544 & & 0.538 \\
\hline $\mathrm{T} 1$ & 21 & $35(2.7-150)$ & & $2.1(0.19-27)$ & & $6.5(0.2-33.6)$ & & $12.6(4.6-45.2)$ & \\
\hline $\mathrm{T} 2$ & 25 & $7.8(1.1-135)$ & & $12(4.7-45.3)$ & & $4.6(1.1-11.3)$ & & $12.3(1.4-44.2)$ & \\
\hline T3 & 14 & $244(16-985)$ & & $49(3.7-86.4)$ & & $8.3(1.1-61.8)$ & & $10.5(1.6-17.9)$ & \\
\hline LN: & & & 0.242 & & $0.047^{*}$ & & 0.739 & & 0.443 \\
\hline Negative & 39 & $20(1.6-152)$ & & $7.5(0.2-36.6)$ & & $5.1(0.51-23)$ & & $12.6(1.5-44.3)$ & \\
\hline Positive & 21 & $39(6.9-289)$ & & $29.2(3.8-64)$ & & $6.2(1.0-30.1)$ & & $7.9(1.5-27.7)$ & \\
\hline Recurrence: & & & $0.003^{*}$ & & $0.035^{*}$ & & 0.285 & & 0.144 \\
\hline Negative & 44 & $13(1.4-130)$ & & $6.1(0.6-45.7)$ & & $4.6(0.3-19)$ & & $11.4(1.4-35)$ & \\
\hline Positive & 16 & $236(34-1708)$ & & $36.2(17-60.4)$ & & $12.4(1.3-38)$ & & $23.7(4-61)$ & \\
\hline PFS: & & & $0.001^{*}$ & & $0.004^{\star}$ & & $0.048^{*}$ & & $0.009^{*}$ \\
\hline$\leq 12 \mathrm{~m}$ & 22 & $281(55-1168)$ & & $37.6(13.9-64)$ & & $14(3.7-61.8)$ & & $17.3(3.9-50.5)$ & \\
\hline$\leq 24 \mathrm{~m}$ & 31 & $14.6(1.6-61)$ & & $6.8(0.2-446)$ & & $4.6(0.19-12)$ & & $15.2(1.3-39)$ & \\
\hline$\leq 36 \mathrm{~m}$ & 7 & $1.5(0.7-7.8)$ & & $3.5(1.05-4.1)$ & & $2.7(1.5-10.2)$ & & $1.4(0.3-1.5)$ & \\
\hline OS: & & & $0.001^{*}$ & & $0.011^{*}$ & & $0.009^{*}$ & & $0.009^{*}$ \\
\hline$\leq 12 \mathrm{~m}$ & 19 & $456(123-1978)$ & & $36.6(12-65)$ & & $306(4.6-61)$ & & $15.2(4.1-49)$ & \\
\hline$\leq 24 \mathrm{~m}$ & 34 & $22.4(1.6-69)$ & & $7.5(0.2-48)$ & & $3.9(0.1-12)$ & & $16.3(1.2-40)$ & \\
\hline$\leq 36 \mathrm{~m}$ & 7 & $1.5(0.7-7.8)$ & & $3.5(1.1-4.1)$ & & $2.7(1.5-10)$ & & $1.4(0.3-1.5)$ & \\
\hline
\end{tabular}

Data are presented as median (quartiles). HPD - histopathological diagnosis, CCRCC - clear cell renal cell carcinoma, pRCC - papillary cell RCC, chRCC - chromophobe RCC, $T$ - tumour size, LN - lymph node, PFS - progressive-free survival, OS - overall survival. Mann-Whitney U and Kruskal-Wallis tests were used. *Values are statistically significant at $p<0.05$.

dence interval $(\mathrm{Cl}): 1.13-15.6, p=0.031 ; \mathrm{HR}=3.80$, 95\% Cl: $1.55-9.30, p=0.003$; and $\mathrm{HR}=4.94$, 95\% Cl: 1.98-12.3, $p=0.001$; respectively) in RCC patients. Using Kaplan-Meier analysis, a comparison of survival curves of patients with low versus high LINC-ROR, SOX2, NANOG, and POU5F1 gene expression revealed significant differences in OS ( $p=0.018,0.008,0.028$, and 0.001 , respectively), which suggested that high expression was associated with poor survival (Figure 4). Similar find- 
Table IV. Correlation analysis between gene expression and the clinicopathological characteristics in RCC patients

\begin{tabular}{|lcccccccc|}
\hline \multirow{2}{*}{ Parameter } & \multicolumn{2}{c}{ LINC-ROR } & \multicolumn{2}{c}{ SOX2 } & \multicolumn{2}{c|}{ NANOG } & \multicolumn{2}{c|}{ POU5F1 } \\
\cline { 2 - 9 } & $r$ & $P$-value & $r$ & $P$-value & $r$ & $P$-value & $r$ & $P$-value \\
\hline Age & -0.002 & 0.987 & -0.189 & 0.148 & 0.009 & 0.949 & -0.031 & 0.812 \\
\hline Gender & -0.245 & 0.059 & 0.065 & 0.624 & -0.021 & 0.872 & -0.064 & 0.629 \\
\hline HPD & 0.076 & 0.563 & 0.045 & 0.733 & 0.031 & 0.816 & -0.053 & 0.687 \\
\hline Side & 0.014 & 0.916 & -0.043 & 0.745 & -0.045 & 0.733 & 0.194 & 0.138 \\
\hline Number & 0.100 & 0.447 & 0.190 & 0.146 & 0.134 & 0.306 & -0.092 & 0.484 \\
\hline Grade & 0.387 & $\mathbf{0 . 0 0 2}$ & 0.314 & $\mathbf{0 . 0 1 4}$ & 0.265 & $\mathbf{0 . 0 4 1}$ & 0.077 & 0.558 \\
\hline Tumour size & 0.185 & 0.158 & 0.304 & $\mathbf{0 . 0 1 8}$ & -0.009 & 0.946 & -0.141 & 0.282 \\
\hline LN & 0.152 & 0.245 & 0.258 & $\mathbf{0 . 0 4 6}$ & 0.043 & 0.742 & -0.100 & 0.448 \\
\hline Recurrence & 0.383 & $\mathbf{0 . 0 0 3}$ & 0.274 & $\mathbf{0 . 0 3 4}$ & 0.139 & 0.289 & 0.190 & 0.145 \\
\hline PFS & -0.577 & $<\mathbf{0 . 0 0 1}$ & -0.511 & $<0.001$ & -0.339 & $\mathbf{0 . 0 0 8}$ & -0.419 & $\mathbf{0 . 0 0 1}$ \\
\hline OS & -0.576 & $<\mathbf{0 . 0 0 1}$ & -0.488 & $<0.001$ & -0.403 & $\mathbf{0 . 0 0 1}$ & -0.464 & $<0.001$ \\
\hline
\end{tabular}

Data are shown as a correlation coefficient $(r)$ and its $p$ value. HPD - histopathological diagnosis, LN - lymph node, PFS - progressive-free survival, OS - overall survival. Spearman's rank correlation test was used. Bold values are statistically significant at $p<0.05$.

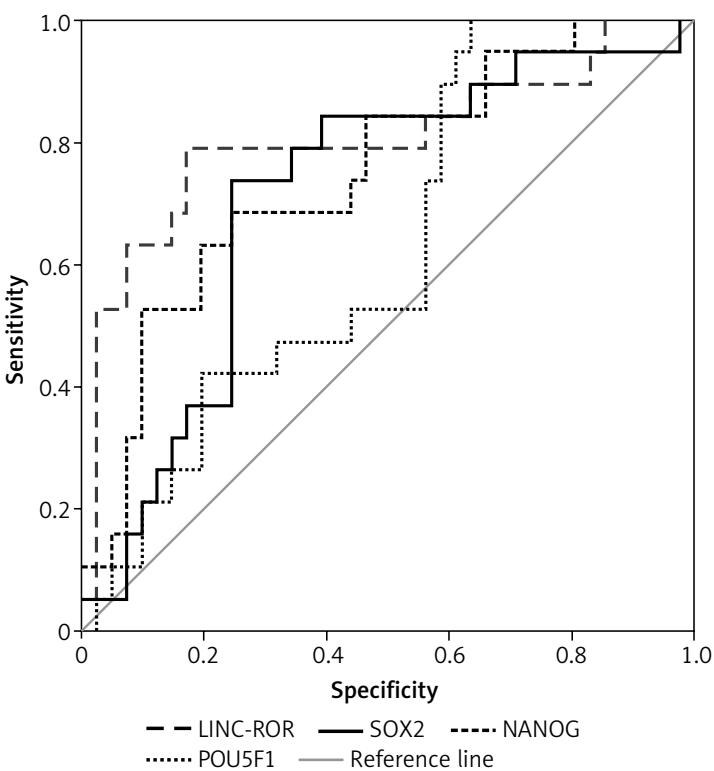

Figure 3. ROC analysis of RCC patients

ings were found in samples stratified by the histopathological type.

\section{Multivariate analysis}

Bray-Curtis analysis was applied to combine the effects of both clinicopathological characteristics and the transcriptomic signatures of the four tested genes. Samples were scattered along multiple axes; axis 1 represented $30.1 \%$ of the variance, whereas axes 2 and 3 accounted for $14.6 \%$ and $11 \%$ of clustering, respectively. The study population was clustered into three distinct groups according to survival time (Figure 5). Notably, patients with poor survival (i.e. less than 1 year) were most affected by high LINC-ROR levels. The same category of patients was associated with poor differentiation and recurrence.

\section{Discussion}

A complex network of TFs, non-coding RNAs, and signalling transducers drives cancer. Unravelling the interactions between these molecular cancer players would pave the road towards a better understanding of cancer development and progression.

Currently, marked up-regulation of LINC-ROR, SOX2, NANOG, and POU5F1 genes was found in cancer samples compared with the adjacent non-cancer tissues. Additionally, LINC-ROR expression levels were positively correlated with SOX2 and NANOG levels. These results were consistent with previous studies [14, 15]. Pluripotent TFs, which are often epigenetically maintained in a silent state, have been shown to be reactivated in several cancers, and their knockdown or suppression with microRNA or small interfering RNA transfection has been shown to hinder tumour progression, promote apoptosis, and improve chemosensitivity [21]. Interestingly, these TFs are now well known to operate in conjunction with non-coding RNAs. A few human IncRNAs may also be under the direct control of the core pluripotency TFs [22]. A recently identified cytoplasmic IncRNA, LINC-ROR, was reported to play a crucial role in the pluripotency maintenance [23]. The promoter of the LINC-ROR gene was found to contain binding sites for SOX2, NANOG, and POU5F1 [24]. Upon binding of TFs, LINC-ROR transcription is activated, whereas silencing of these proteins suppressed LINC-ROR expression through a regulatory feedback loop [25]. Similarly to our findings, LINC-ROR over-expression has also been observed in several cancers, including colorectal carcinoma [15] and breast cancer [25].

Another main finding of our study was the association of the four genes with poor prognosis. The 
Table V. The prognostic performance of single and joint detection of genes in renal cell carcinoma to predict survival time

\begin{tabular}{|llc|}
\hline Parameter & Area $(95 \% \mathrm{Cl})$ & $P$-value \\
\hline Single gene: & $0.804(0.667-0.941)$ & $<0.001^{*}$ \\
\hline LINC-ROR & $0.714(0.573-0.854)$ & $0.008^{*}$ \\
\hline SOX2 & $0.748(0.615-0.882)$ & $0.002^{*}$ \\
\hline NANOG & $0.632(0.488-0.775)$ & 0.103 \\
\hline POU5F1 & $0.748(0.652-0.845)$ & $<0.001^{*}$ \\
\hline LINC-ROR + SOX2 & $0.755(0.659-0.850)$ & $<0.001^{*}$ \\
\hline LINC-ROR + NANOG & $0.715(0.616-0.814)$ & $<0.001^{*}$ \\
\hline LINC-ROR + POU5F1 & $0.735(0.640-0.831)$ & $0.001^{*}$ \\
\hline SOX2 + NANOG & $0.675(0.576-0.773)$ & $0.001^{*}$ \\
\hline SOX2 + POU5F1 & $0.695(0.599-0.791)$ & $<0.001^{*}$ \\
\hline NANOG + POU5F1 & $0.743(0.665-0.821)$ & $<0.001^{*}$ \\
\hline LINC-ROR + SOX2 + NANOG & $0.711(0.632-0.791)$ & $<0.001^{*}$ \\
\hline LINC-ROR + SOX2 + POU5F1 & $0.720(0.641-0.798)$ & $<0.001^{*}$ \\
\hline LINC-ROR + NANOG + POU5F1 & $0.703(0.624-0.781)$ & $<1^{*}$ \\
\hline SOX2 + NANOG + POU5F1 & $0.719(0.651-0.786)$ & \\
\hline LINC-ROR + SOX2 + NANOG +POU5F1 & & \\
\hline
\end{tabular}

Under the non-parametric assumption. *Values indicate significance at $p<0.05$.

Table VI. Multivariable analysis using logistic regression test to predict post-operative recurrence in renal cell carcinoma

\begin{tabular}{|lccccccc|}
\hline Parameter & B & SE & Wald & P-value & OR & \multicolumn{2}{c|}{$95 \% \mathrm{CI}$} \\
\cline { 5 - 8 } & & & & & & & Lower bound Upper bound \\
\hline LINC-ROR & 0.001 & 0.000 & 3.938 & $0.047^{*}$ & 1.001 & 1.000 & 1.002 \\
\hline SOX2 & -0.003 & 0.006 & 0.263 & 0.608 & 0.997 & 0.986 & 1.008 \\
\hline NANOG & 0.000 & 0.010 & 0.002 & 0.961 & 1.000 & 0.981 & 1.019 \\
\hline POU5F1 & 0.000 & 0.000 & 0.088 & 0.767 & 1.000 & 1.000 & 1.000 \\
\hline Age & -0.104 & 0.062 & 2.781 & $0.095^{*}$ & 0.901 & 0.798 & 1.018 \\
\hline Gender & 0.047 & 0.795 & 0.004 & 0.953 & 1.048 & 0.221 & 4.983 \\
\hline LN metastasis & 2.137 & 0.796 & 7.207 & $0.007^{*}$ & 8.470 & 1.780 & 40.300 \\
\hline Constant & 4.016 & 3.760 & 1.141 & 0.285 & 55.467 & & \\
\hline
\end{tabular}

LINC-ROR - long intergenic non-coding RNA-regulator of reprogramming, SOX2, SRY (sex determining region Y)-box 2, NANOG - named after Celtic word Tír na nÓg meaning the land of the young, POU5F1 domain, class 5, transcription factor 1, LN-lymph node, SE - standard error, $\mathrm{OR}$ - odds ratio, $\mathrm{Cl}$-confidence interval. *Values indicate significance at $p<0.05$.

Kaplan-Meier curves and ROC analyses showed that the LINC-ROR, SOX2, NANOG, and POU5F1 expression profiles were associated with shorter survival times. RCC patients with high LINC-ROR and SOX2 levels had a higher recurrence rate after an average of 4 months post-nephrectomy. Notably, regression analyses confirmed that LINCROR was an independent predictor for recurrence and poor OS. In addition, LINC-ROR, SOX2, and NANOG expression showed a significant association with poor differentiation, whereas SOX2 was a poor marker for LN metastasis. In accordance with previous studies, melanoma and HCC cells expressing high POU5F1 and NANOG levels ex- hibited a more aggressive malignant phenotype [26]. NANOG expression has also been considered a prognostic biomarker for triple-negative breast cancer (TNBC) [27].

Recently, increasing attention has been focused on the contribution of LINC-ROR to cancer progression. LINC-ROR has been suggested to be a driving factor in tumourigenesis [24], as depicted in Figure 6. Increased LINC-ROR expression levels have been associated with differentiation, apoptosis, invasion, EMT, and metastasis in cancer $[24,28]$. Up-regulation was recognised as a poor prognostic factor in colon cancer [29]. However, LINC-ROR silencing reduced the malig- 
Table VII. Survival analysis of renal cell carcinoma patients

\begin{tabular}{|c|c|c|c|c|c|c|c|}
\hline \multirow[t]{2}{*}{ Parameter } & \multirow{2}{*}{$\begin{array}{l}\text { Survival time, } \\
\text { median }(95 \% \mathrm{Cl}) \\
\text { [months] }\end{array}$} & \multicolumn{3}{|c|}{ Overall comparisons } & \multicolumn{3}{|c|}{ Cox regression } \\
\hline & & $\begin{array}{l}\text { Log } \\
\text { rank }\end{array}$ & Breslow & $\begin{array}{l}\text { Tarone- } \\
\text { Ware }\end{array}$ & HR $(95 \% \mathrm{Cl})$ & Wald & $P$-value \\
\hline \multicolumn{8}{|l|}{ Age: } \\
\hline$<60$ years & $13(9.8-6.1)$ & 0.088 & 0.054 & 0.061 & Ref. & & \\
\hline$\geq 60$ years & $17(14.9-19.0)$ & & & & $0.98(0.94-1.03)$ & 0.317 & 0.573 \\
\hline \multicolumn{8}{|l|}{ Gender: } \\
\hline Female & $13(8.51-17.4)$ & 0.127 & 0.077 & 0.095 & Ref. & & \\
\hline Male & $16(13.3-18.6)$ & & & & $0.59(0.26-1.33)$ & 1.608 & 0.205 \\
\hline \multicolumn{8}{|l|}{ HPD: } \\
\hline $\mathrm{cCRCC}$ & $15(12.3-17.6)$ & 0.103 & 0.198 & 0.159 & Ref. & & \\
\hline $\mathrm{pRCC}$ & $17(13.2-20.7)$ & & & & $0.60(0.25-1.44)$ & 1.307 & 0.253 \\
\hline chRCC & $15(7.42-22.5)$ & & & & $1.41(0.62-3.11)$ & 0.740 & 0.390 \\
\hline \multicolumn{8}{|l|}{ Side: } \\
\hline Right & $16(12.3-19.6)$ & 0.703 & 0.489 & 0.539 & Ref. & & \\
\hline Left & $15(12.9-17.0)$ & & & & $1.06(0.54-208)$ & 0.034 & 0.854 \\
\hline \multicolumn{8}{|l|}{ Number: } \\
\hline Unifocal & $16(14.3-17.6)$ & 0.461 & 0.291 & 0.350 & Ref. & & \\
\hline Multifocal & $13(11.1-14.8)$ & & & & $0.89(0.38-2.10)$ & 0.063 & 0.802 \\
\hline \multicolumn{8}{|l|}{ Grade: } \\
\hline 1 & $16(13.1-189)$ & $0.005^{*}$ & $0.003^{*}$ & $0.003^{*}$ & Ref. & & \\
\hline 2 & $18(15.4-20.5)$ & & & & $0.47(0.17-1.30)$ & 2.101 & 0.147 \\
\hline 3 & $12(8.4-155)$ & & & & $0.82(0.22-2.98)$ & 0.089 & 0.765 \\
\hline \multicolumn{8}{|l|}{$\mathrm{T}:$} \\
\hline $\mathrm{T} 1$ & $16(11.5-20.4)$ & 0.709 & 0.577 & 0.635 & Ref. & & \\
\hline $\mathrm{T} 2$ & $16(12.7-19.2)$ & & & & $0.84(0.40-1.77)$ & 0.195 & 0.659 \\
\hline T3 & $13(9.3-16.6)$ & & & & $0.93(0.26-3.34)$ & 0.011 & 0.917 \\
\hline \multicolumn{8}{|l|}{ LN: } \\
\hline Negative & $16(13.5-18.4)$ & 0.306 & 0.235 & 0.255 & Ref. & & \\
\hline Positive & $14(11.3-16.6)$ & & & & $0.83(0.23-2.89)$ & 0.084 & 0.772 \\
\hline \multicolumn{8}{|l|}{ Recurrence: } \\
\hline Negative & $17(15.2-18.7)$ & $<0.001^{*}$ & $<0.001^{*}$ & $<0.001^{*}$ & Ref. & & \\
\hline Positive & $10(9.1-10.9)$ & & & & $4.94(1.98-12.3)$ & 11.782 & $0.001^{*}$ \\
\hline \multicolumn{8}{|l|}{ LINC-ROR: } \\
\hline Low & $22(13.5-30.4)$ & $0.007^{*}$ & $0.018^{*}$ & $0.012^{*}$ & Ref. & & \\
\hline High & $15(12.9-17.0)$ & & & & $4.21(1.13-15.6)$ & 4.641 & $0.031^{*}$ \\
\hline \multicolumn{8}{|l|}{ SOX2: } \\
\hline Low & $20(16.6-23.3)$ & $0.089^{*}$ & $0.008^{*}$ & $0.018^{*}$ & Ref. & & \\
\hline High & $14(12.1-15.8)$ & & & & $2.57(0.99-6.6)$ & 3.814 & 0.051 \\
\hline \multicolumn{8}{|l|}{ NANOG: } \\
\hline Low & $18(16.7-19.2)$ & 0.295 & $0.028^{*}$ & 0.081 & Ref. & & \\
\hline High & $14(11.8-16.1)$ & & & & $1.10(0.50-2.41)$ & 0.065 & 0.799 \\
\hline \multicolumn{8}{|l|}{ POU5F1: } \\
\hline Low & $22(19.1-24.8)$ & $0.003^{*}$ & $0.001^{*}$ & $0.001^{*}$ & Ref. & & \\
\hline High & $14(12.1-15.8)$ & & & & $3.80(1.55-9.30)$ & 8.544 & $0.003^{*}$ \\
\hline
\end{tabular}



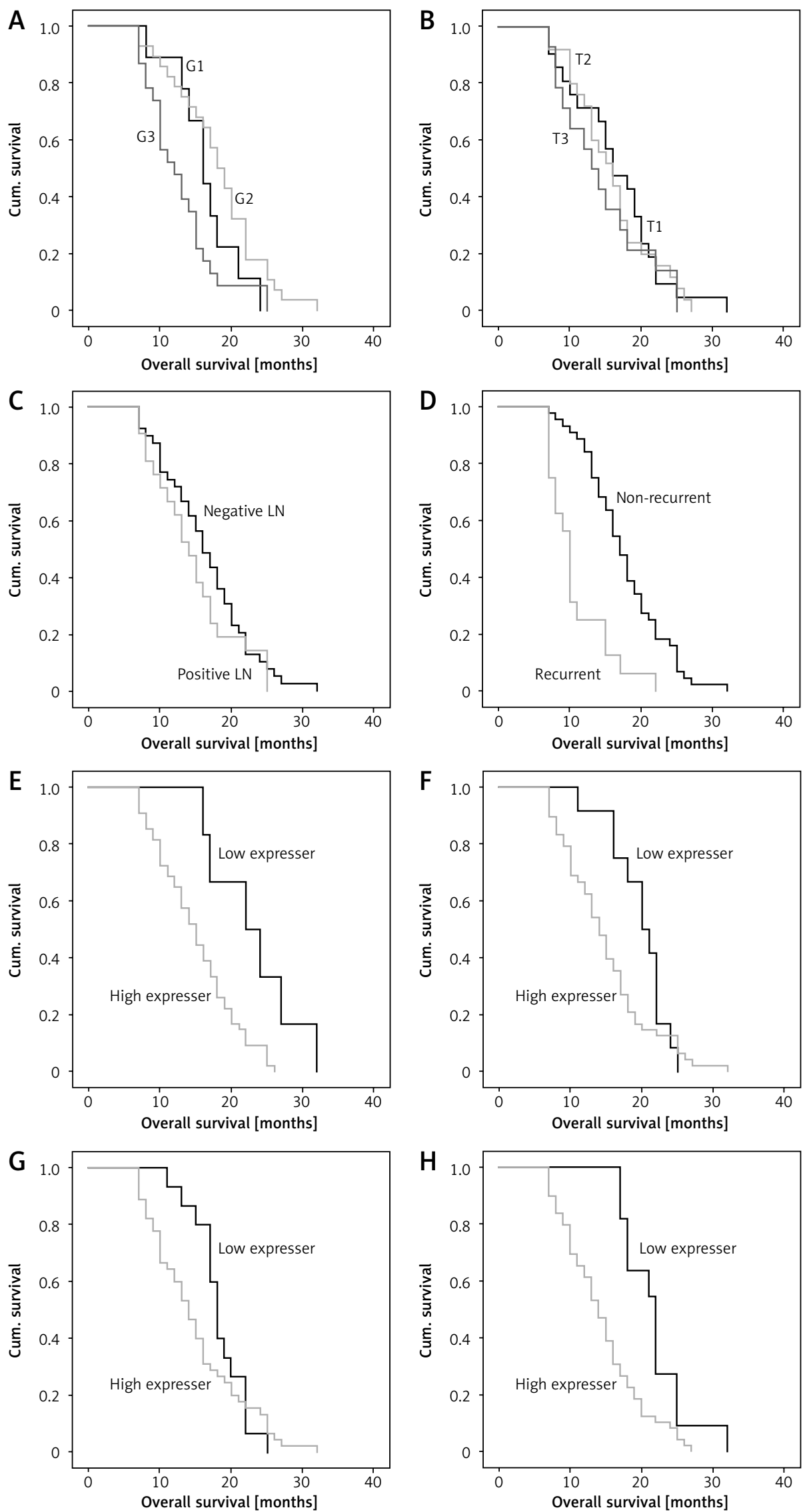

Figure 4. Kaplan-Meier curves comparing patient survival with different clinical variables (A - tumor grade, B - tumor size, C - LN infiltration, D - recurrence, E - LINC-ROR, F - SOX2, G - NANOG, H - POU5F1). Log-rank (Mantel-Cox) test was used for comparisons. The significance level was set at $p<0.05$ 


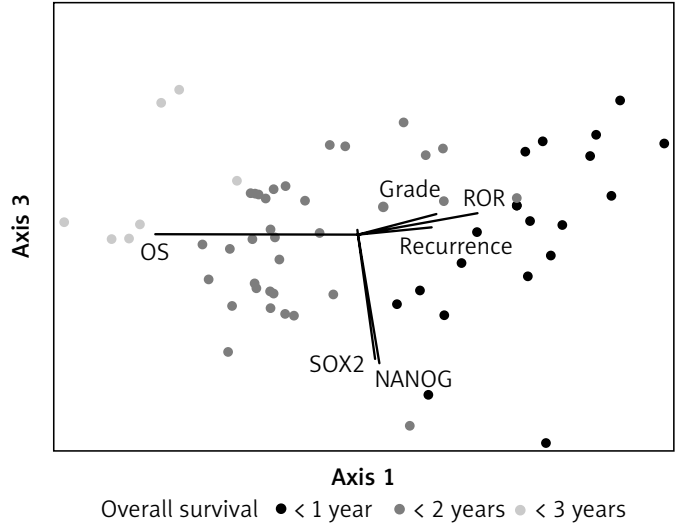

Figure 5. Multivariate analysis cluster of RCC patients according to the combined transcriptomic gene signature and clinicopathological features. PC-ORD v5.0 was used for data exploration. Ordination graph constructed via Bray-Curtis analysis. The following setup parameters were adjusted. Distance method: relative Euclidean method; endpoint selection method: variance-regression; axis projection geometry: Euclidean; residual distances: Euclidean; score calculation by weighted averaging. Samples were scattered along multiple axes; axis 1 represents $30.1 \%$ of variance, whereas axes 2 and 3 account for $14.6 \%$ and $11 \%$ of clustering, respectively. The study population was clustered into three distinct groups according to survival time. Notably, most of the patients with poor survival (less than 1 year) manifested high levels of LINCROR. The same category of patients was associated with poor differentiation and recurrence

nant phenotype of cancer cells [24]. One of the peculiar roles of IncRNAs is acting as competing endogenous RNAs (ceRNAs) by binding and se- questering microRNAs (miRNAs) and thus preventing miRNAs from silencing their target genes [30], a process that has been shown to be relevant to tumourigenesis and differentiation [12]. LINC-ROR has been demonstrated to serve as an miRNA sponge in embryonic stem cell self-renewal [31]. LINC-ROR effectively maintained the levels of the SOX2 TF by binding to miR-145 in human amniotic epithelial stem cells, altering the landscape of pluripotency and differentiation [32]. The tumour-promoting function of LINC-ROR can also be attributed to its influence on transcription frameworks involved in various cancer-related signalling pathways [24]. LINC-ROR can play an oncogenic role by modulating the c-Myc pathway [20]. LINC-ROR has also been shown to elicit re-expression of foetal genes as atrial and brain natriuretic peptides [28]. Additionally, upon DNA damage and expression of the tumour suppressor Tp53, LINC-ROR levels increased and suppressed Tp53 mRNA through a translation repression mechanism [16]. At the DNA level, LINC-ROR has been reported to block the recruitment of a histone-modifying enzyme (G9A methyltransferase), thereby triggering chromatin modification and promoting tumour growth and metastasis [32].

In conclusion, deregulation of the LINC-ROR/ pluripotent gene axis in renal tumours highlights their use as potential prognostic biomarkers. Targeting this RNA directly rather than targeting its regulated miRNAs, tumour suppressor genes, or oncogenes might provide a shortcut for future cancer therapies. However, due to limited clini-

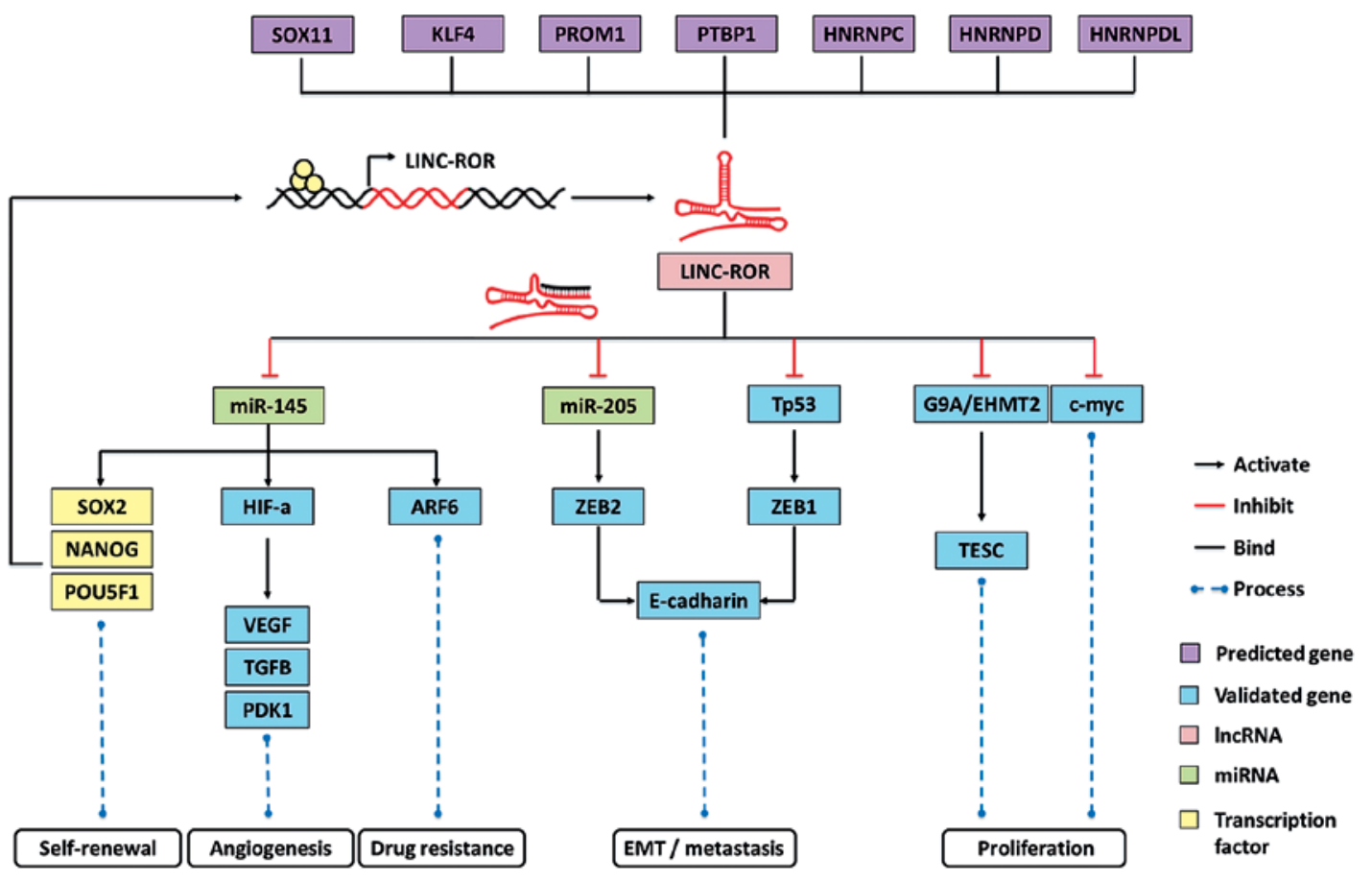

Figure 6. The role of LINC-ROR in tumourigenesis and cancer progression 
cal studies on LINC-ROR, further studies in other types of tumours are warranted.

\section{Acknowledgments}

The authors thank the Centre of Excellence in Molecular and Cellular Medicine and Oncology Diagnostic Unit for providing the facilities for work.

\section{Conflict of interest}

The authors declare no conflict of interest.

\section{References}

1. Bhatt JR, Finelli A. Landmarks in the diagnosis and treatment of renal cell carcinoma. Nat Rev Urol 2014; 11: 517-25.

2. Posadas EM, Limvorasak S, Figlin RA. Targeted therapies for renal cell carcinoma. Nat Rev Nephrol 2017; 13: 496-511.

3. Hsieh JJ, Purdue MP, Signoretti S, et al. Renal cell carcinoma. Nat Rev Dis Primers 2017; 3: 17009.

4. Zhao JJ, Chen PJ, Duan RQ, Li KJ, Wang YZ, Li Y. miR-630 functions as a tumor oncogene in renal cell carcinoma. Arch Med Sci 2016; 12: 473-8.

5. Jędroszka D, Orzechowska M, Bednarek AK. Predictive values of Notch signalling in renal carcinoma. Arch Med Sci 2017; 13: 1249-54.

6. Lin VTG, Pruitt HC, Samant RS, Shevde LA. Developing cures: targeting ontogenesis in cancer. Trends Cancer 2017; 3: 126-36.

7. Goding CR, Pei D, Lu X. Cancer: pathological nuclear reprogramming? Nat Rev Cancer 2014; 14: 568-73.

8. Hnisz D, Schuijers J, Lin CY, et al. Convergence of developmental and oncogenic signaling pathways at transcriptional super-enhancers. Mol Cell 2015; 58: 362-70.

9. Liu A, Yu X, Liu S. Pluripotency transcription factors and cancer stem cells: small genes make a big difference. Chin J Cancer 2013; 32: 483-7.

10. Alderton GK. Pluripotency: partial reprogramming induces cancer. Nat Rev Cancer 2014; 14: 216-7.

11. Loewer S, Cabili MN, Guttman M, et al. Large intergenic non-coding RNA-RoR modulates reprogramming of human induced pluripotent stem cells. Nature Genet 2010; 42: 1113-7.

12. Fatica A, Bozzoni I. Long non-coding RNAs: new players in cell differentiation and development. Nat Rev Genet 2014; 15: 7-21.

13. Guttman M, Donaghey J, Carey BW, et al. lincRNAs act in the circuitry controlling pluripotency and differentiation. Nature 2011; 477: 295-30.

14. Sheik Mohamed J, Gaughwin PM, Lim B, Robson P, Lipovich L. Conserved long noncoding RNAs transcriptionally regulated by Oct4 and Nanog modulate pluripotency in mouse embryonic stem cells. RNA 2010; 16: 324-37.

15. Li H, Jiang X, Niu X. Long non-coding RNA reprogramming (ROR) promotes cell proliferation in colorectal cancer via affecting P53. Med Sci Monit 2017; 23: 919-28.

16. Zhang A, Zhou N, Huang J, et al. The human long noncoding RNA-RoR is a p53 repressor in response to DNA damage. Cell Res 2013; 23: 340-50.

17. Li Y, Jiang B, Zhu H, et al. Inhibition of long non-coding RNA ROR reverses resistance to Tamoxifen by inducing autophagy in breast cancer. Tumour Biol 2017; 39: 1010428317705790.
18. Toraih EA, Ibrahiem AT, Fawzy MS, Hussein MH, Al-Qahtani SAM, Shaalan AAM. MicroRNA-34a: a key regulator in the hallmarks of renal cell carcinoma. Oxid Med Cell Longev 2017; 2017: 3269379.

19. Toraih EA, Fawzy MS, El-Falouji Al, et al. Stemness-related transcriptional factors and homing gene expression profiles in hepatic differentiation and cancer. Mol Med 2016; 22: 653-63.

20. Huang J, Zhang A, Ho TT, et al. Linc-RoR promotes c-Myc expression through hnRNP I and AUF1. Nucleic Acids Res 2016; 44: 3059-69.

21. Yu AQ, Ding Y, Li CL. TALEN-induced disruption of Nanog expression results in reduced proliferation, invasiveness and migration, increased chemosensitivity and reversal of EMT in HepG2 cells. Oncol Rep 2016; 35: 1657-63.

22. Ng SY, Johnson R, Stanton LW. Human long non-coding RNAs promote pluripotency and neuronal differentiation by association with chromatin modifiers and transcription factors. EMBO I 2012; 31: 522-33.

23. Rosa A, Ballarino M. Long noncoding RNA regulation of pluripotency. Stem Cells Int 2016; 2016: 1797692.

24. Pan Y, Li C, Chen J, et al. The emerging roles of long noncoding RNA ROR (lincRNA-ROR) and its possible mechanisms in human cancers. Cell Physiol Biochem 2016; 40: 219-29.

25. Tordonato C, Di Fiore PP, Nicassio F. The role of non-coding RNAs in the regulation of stem cells and progenitors in the normal mammary gland and in breast tumors. Front Genet 2015; 6: 72.

26. Shan J, Shen J, Liu L, et al. Nanog regulates self-renewal of cancer stem cells through the insulin-like growth factor pathway in human hepatocellular carcinoma. Hepatology 2012; 56: 1004-14.

27. Nagata T, Shimada Y, Sekine S, et al. KLF4 and NANOG are prognostic biomarkers for triple-negative breast cancer. Breast Cancer 2017; 24: 326-35.

28. Arunkumar G, Deva Magendhra Rao AK, Manikandan M, et al. Expression profiling of long non-coding RNA identifies linc-RoR as a prognostic biomarker in oral cancer. Tumour Biol 2017; 39: 1010428317698366.

29. Zhou P, Sun L, Liu D, Liu C, Sun L. Long non-coding RNA lincRNA-ROR promotes the progression of colon cancer and holds prognostic value by associating with miR-145. Pathol Oncol Res 2016; 22: 733-40.

30. Wang $Y, X u Z$, Jiang J, et al. Endogenous miRNA sponge lincRNA-RoR regulates Oct4, Nanog, and Sox2 in human embryonic stem cell self-renewal. Dev Cell 2013; 25: 69-80.

31. Cheng EC, Lin H. Repressing the repressor: a lincRNA as a microRNA sponge in embryonic stem cell self-renewal. Dev Cell 2013; 25: 1-2.

32. Fan J, Xing $Y$, Wen $X$, et al. Long non-coding RNA ROR decoys gene-specific histone methylation to promote tumorigenesis. Genome Biol 2015; 16: 275. 